\title{
Effect of seed bacterization on plant growth response and induction of disease resistance in chilli
}

\begin{abstract}
This study aimed to examine the induction of disease resistance, and growth response in chilli plants elicited by plant growth promoting endophytic bacteria [Pseudomonas aeruginosa (UPMP3), Burkholderia cepacia (UPMB3), and Serratia marcescens (UPMS3)]. Seed bacterization with UPMP3 and UPMB3 significantly increased peroxidase (PO), polyphenol oxidase (PPO), and phenylalanine ammonia-lyase (PAL) activities. This increase corresponded to greater reduction in pre- and post-emergence damping-off caused by Sclerotium rolfsii. UPMS3 alone or as mixture with UPMP3 and UPMB3 did not show any significant reduction in disease incidence. However, all the isolates tested did not inhibit the seed germination and seedling establishment in chilli.
\end{abstract}

Keyword: Endophytic bacteria; Induced resistance; PO; PPO; PAL; Sclerotium rolfsii 